\title{
ПРО ПОРЯДОК ВИКОРИСТАННЯ ЛІЦЕНЗОВАНОГО ПРОГРАМНОГО ЗАБЕЗПЕЧЕННЯ ДЛЯ РОЗРОБЛЕННЯ МОДЕЛЕЙ КОМП'ЮТЕРНОЇ ГРАФІКИ В МЕДИЧНІЙ ОСВІТІ
}

\author{
В. П. Марценюк, І. Є. Андрущак ${ }^{1}$ \\ Університет Бєльсько-Бяли, Польща \\ ${ }^{1}$ Луцький національний технічний університет
}

\begin{abstract}
У роботі досліджено проблеми, пов'язані з використанням фрізичних моделей у медичній освіті, представлено підхід до розроблення програмного забезпечення з можливістю інтерактивного моделювання анатомічних структур. Використовуване програмне забезпечення орієнтовано на ліцензійну доступність і широке використання в медичних ВНЗах.
\end{abstract}

Ключові слова: комп'ютерна графріка, медична освіта, BodyParts3D, Blender, Unity, 3D-моделювання.

\section{ON APPROACH OF THE LICENSED SOFTWARE USE WITH THE PURPOSE OF COMPUTER GRAPHICS MODELS DEVELOPMENT IN MEDICAL EDUCATION}

V. P. Martsenyuk, I. Ye. Andrushchak ${ }^{1}$

University of Bielsko-Biala, Poland

${ }^{1}$ Lutsk National Technical University

The problems dealing with the use of physical models in medical education are investigated. There is presented the approach for software development with possibility of interactive design of anatomic structures. The used software is oriented to the licensed availability and deployment in medical universities.

Key words: computer graphics, medical education, BodyParts3D, Blender, Unity, 3D-modeling.

\section{О ПОРЯДКЕ ИСПОЛЬЗОВАНИЯ ЛИЦЕНЗИРОВАННОГО ПРОГРАММНОГО ОБЕСПЕЧЕНИЯ С ЦЕЛЬЮ РАЗРАБОТКИ МОДЕЛЕЙ КОМПЬЮТЕРНОЙ ГРАФИКИ В МЕДИЦИНСКОМ ОБРАЗОВАНИИ}

\author{
В. П. Марценюк, И. Е. Андрущак ${ }^{1}$ \\ Университет Бельско-Бялы, Польша \\ ${ }^{1}$ Луцкий национальный технический университет
}

\begin{abstract}
В работе исследованы проблемы, связанные с использованием физических моделей в медицинском образовании, и представлен подход к разработке программного обеспечения с возможностью интерактивного моделирования анатомических структур. Используемое программное обеспечение ориентировано на лицензионную доступность и широкое применение в медицинских вузах.
\end{abstract}

Ключевые слова: компьютерная грасрика, медицинское образование, BodyParts3D, Blender, Unity, 3D-моделирование. 
Введение. Современные подходы к медицинскому образованию требуют активного использования средств интерактивной компьютерной графики и мультимедиа. Так, традиционные учебные методики при изучении морфологических дисциплин концентрируются на использовании учебников, препаратов, двумерных иллюстраций, диаграмм и медицинских изображений. При этом пространственные соотношения сложно интерпретировать без визуализации структур в трёхмерном пространстве (3D) с возможностью интерактивного воздействия. Такие интерактивные учебные материалы позволяют значительно улучшить понимание пространственных соотношений и приобретение знаний $[4,15]$. S. C. Marks [12] утверждает, что человеческое тело - это 3D-объект. Таким образом, изучение анатомии должно полагаться на изучение и оперирование 3D-информацией.

Цель исследования: исследование проблем, связанных с использованием физических моделей в медицинском образовании, и представлении подхода к разработке программного обеспечения с возможностью интерактивного моделирования анатомических структур.

Последние разработки в области программного обеспечения и улучшение качества медицинских изображений позволяют создавать анатомически точные 3D-модели, которые затем могут быть использованы в качестве компьютерных 3D-моделей для обучения. Желательно, чтобы такие учебные модели были наиболее эффективными в комбинации с интерактивным контекстом, таким, как, например, интерактивное обучающее приложение, или при использовании для создания анимаций в курсе анатомии или физиологии [17].

Форма анатомических структур и их пространственные взаимоотношения лучше всего воспринимаются, когда пользователь может взаимодействовать с созданными 3D-моделями, так как в них заложена вся суть 3D-структур и пространственные взаимоотношения; к тому же прилегающие структуры сложно воспринимать другими способами $[4,15]$. Изучая анатомию на человеческих трупах, это можно осуществлять в анатомической лаборатории, хотя для многих материалов (например, кости) для этого существуют ограничения. Профессионально сделанные препараты могут оказаться непрактичными для запоминания и не все образцы развивающихся патологий могут быть доступными, что заставляет студентов учиться «сухо» по учебникам, используя текстовые описания и 2D-изображения. Исследования показывают, что студенты предпочитают учиться, используя одновременно как 2D-изображения, так и интерактивные 3D-модели, а не используя в отдельности 2D- или 3D-информацию [6]. Применение компьютеризированных 3D-моделей позволяет изучать анатомию в любом месте, а не только в анатомической лаборатории или используя препарированные материалы [13]. Сегодня доступен целый ряд анатомических 3D-атласов, разработанных в учебных целях (например, AnatomyBrowser, Zygote Body и BodyParts3D). Все они созданы с использованием данных снимков, полученных с помощью магнитно-резонансной томографии (МРТ) либо компьютерной томографии. Некоторые, включая AnatomyBrowser, имеют возможности хирургического моделирования [2].

Материалы и методы. Физические анатомические модели. Наилучшей моделью для исследований анатомии человека всегда было мёртвое физическое тело [1, 10]. Поскольку при этом в большинстве случаев все части тела правильно расположены, можно прикоснуться ко всем элементам, все структуры (мягкие, твёрдые, гладкие, шероховатые, сухие и влажные) точно воссозданы. С давних времен из-за любопытства люди исследовали раны и органы своих мёртвых сородичей. Однако сегодня доступ к мертвому физическому телу жёстко регулируется правовыми, финансовыми и социальными ограничениями. K тому же, даже при наличии мёртвого физического тела, возникают проблемы с демонстрацией тазовой полости и фасций. И наконец, проблемы донорства мёртвых физических тел, их хранение, использование химических вредных веществ, надлежащее захоронение трупных образцов создают определенные трудности для некоторых учебных заведений.

Для решения таких вопросов используют профессиональные анатомические модели. Древние и современные модели сильно различаются из-за используемых деталей и материалов. Когда-то для представления анатомических структур использовали обычное дерево и слоновую кость, папье-маше или более детальные модели из пластыря, далее были реалистичные восковые модели Susini, Towne, Ziegler и наконец современные профессиональные модели из пластика $[7,11,18]$. Анатомические модели также различаются с точки зрения применения - использование врачами для консультирования пациентов женского пола, 


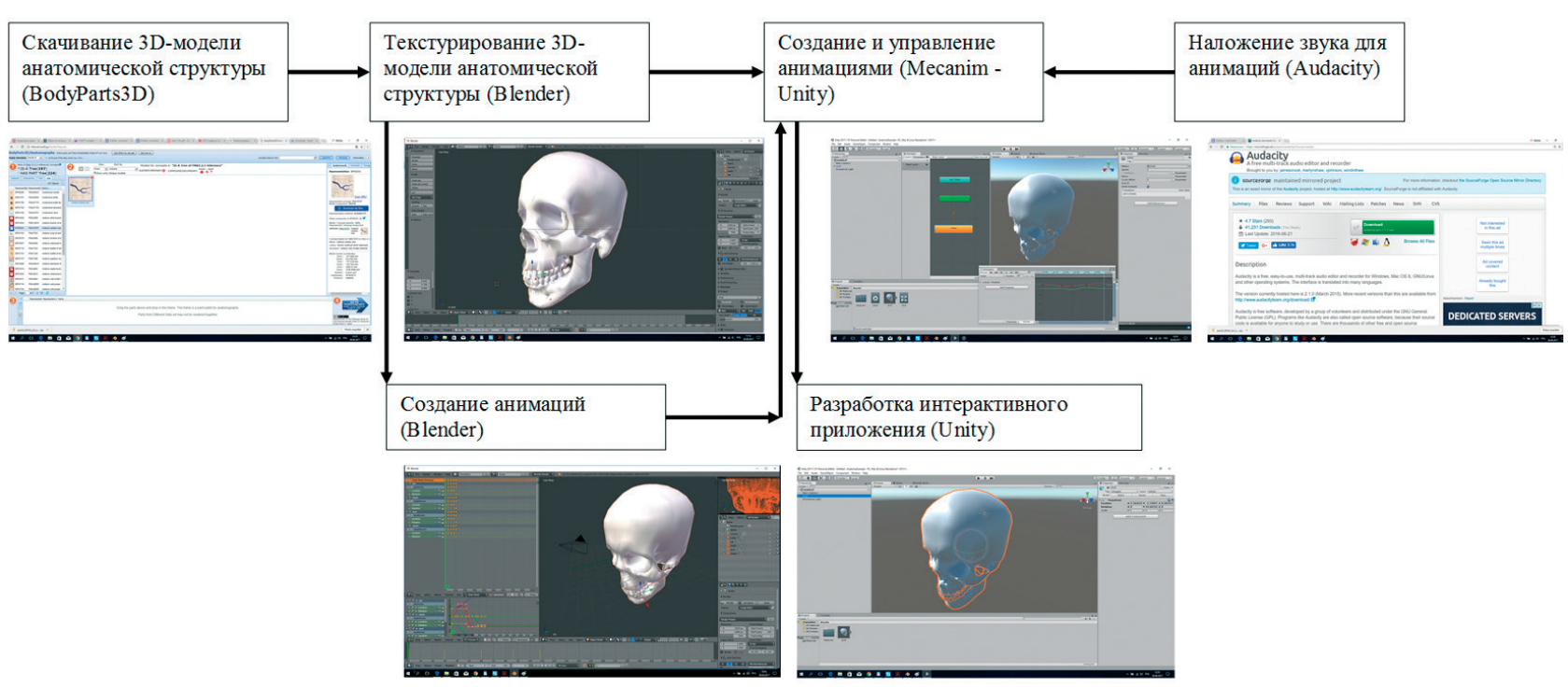

Рис. 1. Порядок разработки интерактивных учебных приложений на основе 3D-моделей

получение навыков хирургами и, конечно, изучение анатомии [9, 14].

Физические анатомические модели обладают свойственными ограничениями при их использовании, хранении и обслуживании. Стоимость профессиональных моделей может достигать тысяч долларов, в зависимости от материала, размера, деталей, точности и интерактивности. К тому же при больших контингентах студентов такие физические анатомические модели могут повреждаться и со временем приходить в негодность. Современные пластические образцы показали свою эффективность при обучении [5, 8]. Однако большинство вышеописанных ограничений присущи также и пластическим образцам.

Цифровые 3D-модели. В дополнение к физическим моделям разработаны современные цифровые 3D-визуализации структур и образцов с использованием медицинских изображений и цифрового 3D-моделирования. Цифровые 3D-модели анатомических структур доступны на компьютере, при использовании мобильных приложений или самостоятельных рабочих станций (например, Anatomage, Touch of Life). Наличие этих материалов в анатомической лаборатории и на учебных компьютерах может снизить потребность в физических анатомических моделях или даже в печатных атласах и поможет преподаванию, предоставив учебные материалы на анатомическом столе. Однако стоимость такой специализированной интерактивной системы 3D-изображений является значительной (примерно 100000 долл. США за стол Anatomage и дополнительные устройства), к тому же следует учитывать потребность постоянного профессионального сопровождения. Использование планшетов iPad и мобильных устройств уменьшает стоимость, но требует разработки специальных приложений, которые тоже влияют на обучение.

Преимущество цифровых 3D-моделей состоит в том, что с ними можно постоянно манипулировать для демонстрации изменений в структуре или образце с учётом возраста и стадии развития; роли хирургической операции либо вмешательства; механизма функционирования. Цифровые 3D-модели способны представить морфогенез сердца или внутреннего уха и прояснить эмбриональные структуры и ткани, являющиеся важными во время успешных стадий эмбриогенеза. К тому же, с помощью цифровых 3D-моделей можно проследить за очагом цирроза печени или прогрессированием болезни Альцгеймера, начиная с начального формирования и до стадии полного разрушения ткани. И наконец, эти модели могут демонстрировать механику функционирования суставов в разных положениях и при нагрузках, либо же физиологию функционирования сердечной мышцы. Напротив, требуется ряд физических моделей для того, чтобы получить любое из таких представлений.

Очевидным преимуществом цифровых анатомических 3D-моделей является то, что преподаватель или учебное учреждение могут создать их за сравнительно короткое время (требуется опытный биомедицинский иллюстратор). Стоимость может 
оказаться относительно небольшой (рабочая станция с программным обеспечением open-source). Однако более эффективное программное обеспечение может быть дорогим (1000-5000 долл. США в год), даже по академической лицензии. Цена лицензии зависит от способа использования (персональное либо для учреждения), переговоров, типа использования (клиническое или учебное). Создание библиотек либо репозиториев моделей может уменьшить потребность в использовании оригинальных моделей и снизить их стоимость [3, 16, 19].

Результаты и их обсуждение. Предлагаемый порядок разработки приложений 3D-моделирования анатомических структур включает следующие этапы работ (рис. 1).

Использование 3D-моделей анатомических структур, разработанных в проекте Anatomography. Проект Anatomography был запущен в 2009 году в Токийском университете (основатель проекта — профессор Коусаку Окубо). База данных сеточных моделей анатомических структур получила название BodyParts3D. Адрес проекта в интернет — http://lifesciencedb.jp/bp3d/?lng=en. Сеточные данные для BodyParts3D были получены на основе MPТ-изображений. Процесс построения моделей для BodyParts3D состоял из трех этапов: 1-й этап: проводилась анатомическая сегментация на основе МРТ-изображений в специальном формате TARO;

2-й этап: с помощью медицинских иллюстраторов, использующих программы 3D-редактирования, добавлялись недостающие детали, делались более чёткими края;

3-й этап: сегментация и модификация данных проводилась в сотрудничестве с клиницистами до тех пор, пока не было достигнуто концептуальное сходство.

Сеточные модели BodyParts3D распространяются в виде файлов формата ОВЈ. Для версии 3.0 полный размер данных составлял 127 МВ (упрощенная сетка) и 521 МВ (высокое качество). При этом количество анатомических структур составляет 1523. Сегодня актуальная версия - 4.0.

Данные в виде архива могут быть загружены из страницы http://dbarchive.biosciencedbc.jp/en/ bodyparts3d/download.html.

Изображения, сгенерированные в Anatomography, и сеточные данные в BodyParts3D имеют лицензию Creative Commons license, Это сделано с целью обеспечения широкого использования и доступности медицинского образования.

Заметим, что анатомические структуры BodyParts3D могут быть интегрированы в более сложную модель, создаваемую, например, в программном обеспечении MakeHuman (рис. 2).

Импортирование и обработка 3D-модели в Blender. Программа Blender предлагает широкий спектр объектов для создания и дальнейшего редактирования: сетки, поверхности NURBS, кривые Безьера, векторные шрифты (TrueTyре, PostScript, OpenType).

Имеется инструментарий для нарезания сетки. Реализованы булевские функции для сеток.

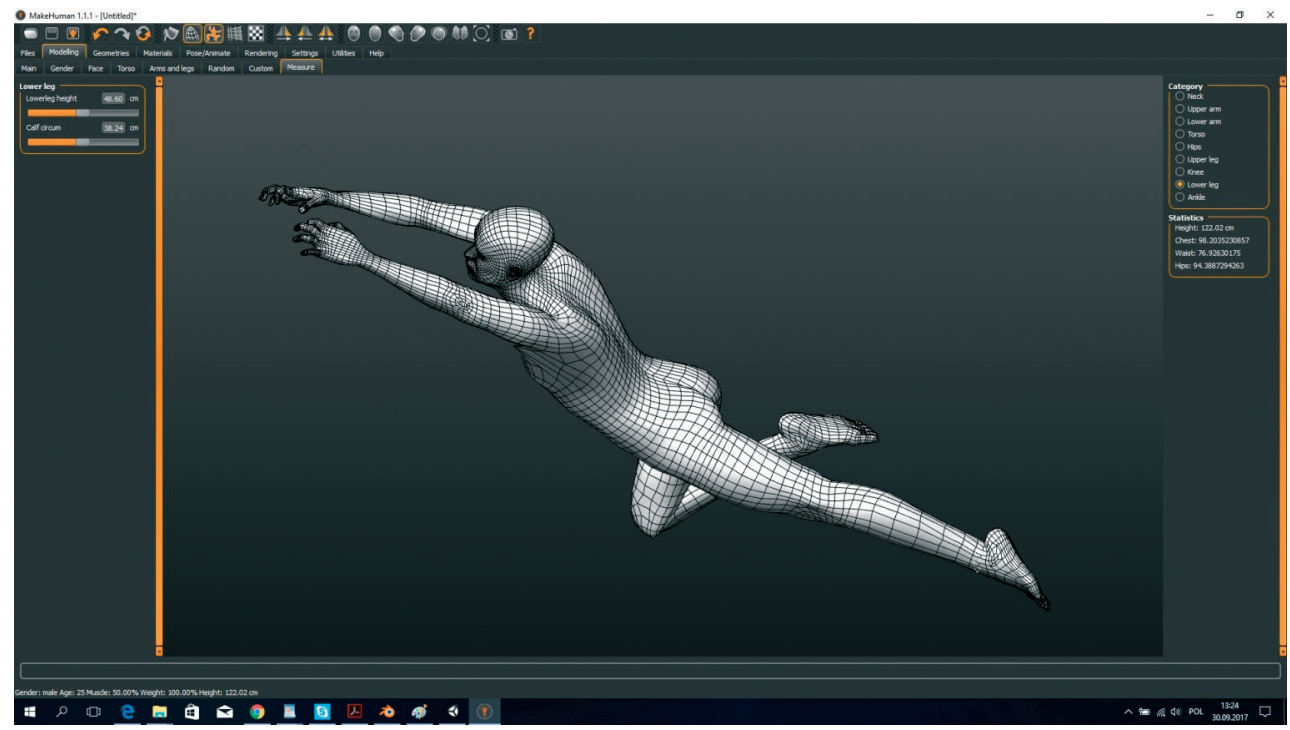

Рис. 2. Программа MakeHuman позволяет создавать сеточные модели в разных положениях 
Редактирование сеток возможно с использованием вершин, рёбер и граней. Существует множество функций для редактирования объектов, которые позволяют получить объект практически любого вида.

С помощью скриптов на языке Python можно создавать новые инструменты пользователя для редактирования.

Blender включает в себя систему BMesh, которая позволяет создавать и редактировать грани с очень сложным строением - например, состоящие из десятков рёбер.

Основными действиями при использовании программы при работе с анатомическими структурами из базы данных BodyParts3D являются:

- импортирование модели в формате ОВЈ-файла (команды File-Import-Wavefront(.obj));

- представление объекта в разных проекциях (виды получаются с помощью клавиш цифровой клавиатуры: для переключения между ортогональной и перспективной проекциями используется клавиша 5, вид спереди - 1, вид справа - 3, вид сверху - 7, вид снизу - 9, поворот окна вида на определённый угол получается при нажатии на клавиши 2, 8, 4, 6, противоположные виды получаются при нажатой клавише Ctrl);

- геометрические преобразования активного объекта (перемещение — клавиша g, обороты клавиша r, масштабирование — клавиша s);

- получение сеточного представления модели (клавиша g);

- переход в режим редактирования и обратно (клавиша Tab);

- редактирование в режимах вершин, рёбер, граней (клавиши нижнего меню);

- наложение материала (созданного на основе текстуры) на соответствующие грани).

Система анимации в Blender основана на использовании прямой и обратной кинематики. Поддерживается автоматический скиннинг, интерактивное рисование распределения весов непосредственно на объекте, ручное редактирование способа обработки каркаса для каждой вершины, система «envelopes».

Реализован миксер нелинейной анимации с циклом передвижения вдоль кривой. При этом возможно анимировать даже отдельные вершины, что позволяет работать даже с очень сложными с точки зрения построения объектами (такими, как анатомические структуры).
Построение анимации основано на использовании системы анимационных кривых, так называемых кривых IPO. Поддерживается принцип «driven-keys», при котором изменение значения одного параметра (например, ширины) приводит к автоматическому изменению (пропорциональному или нет) значения другого (например, высоты). Возможно контролировать значения параметров с помощью математических выражений (написанных на языке Python). Обеспечено использование звуковых файлов и их редактирование с целью соответствующей синхронизации.

Поддерживается синхронизация на основе технологии Motion capture.

K тому же скрипты, написанные на языке Python, создают новые анимационные возможности.

Управление созданием анимации осуществляется с помощью механизма ключей каналов (реализованы каналы перемещения (Location), оборачивания (Rotation), масштабирования (Scaling) и всевозможные комбинации каналов (LocRot, LocScale и т. д.). На основе установленных ключей параметры модели изменяются во всех остальных кадрах на основе автоматически сгенерированных интерполяционных кривых (анимационные кривые), которые имеют графическую визуализацию (рис. 3). Для создания анимации Blender при включённой кнопке записи анимации указатель кадра устанавливается на определённый кадр. Создание ключа канала осуществляется нажатием клавиши і и выбором соответствующей команды контекстного меню.

Созданные таким образом анимации могут далее быть использованы при создании интерактивного приложения.

Создание интерактивного приложения на основе 3D моделей анатомических структур BodyParts3D наиболее целесообразным сегодня представляется с использованием игрового двигателя Unity. Unity, сочетая в себе все свойства современной среды 3D-разработки, используется на основании лицензии Proprietary, которая при использовании для создания компьютерных игр накладывает ограничения на бюджет игры и количество конкурирующих игроков. При этом лицензия Personal с бюджетом игры до 100000 долл. США и количеством конкурирующих игроков до 20 является бесплатной. Разработка интерактивных учебных приложений вполне соответствует таким ограничениям. 

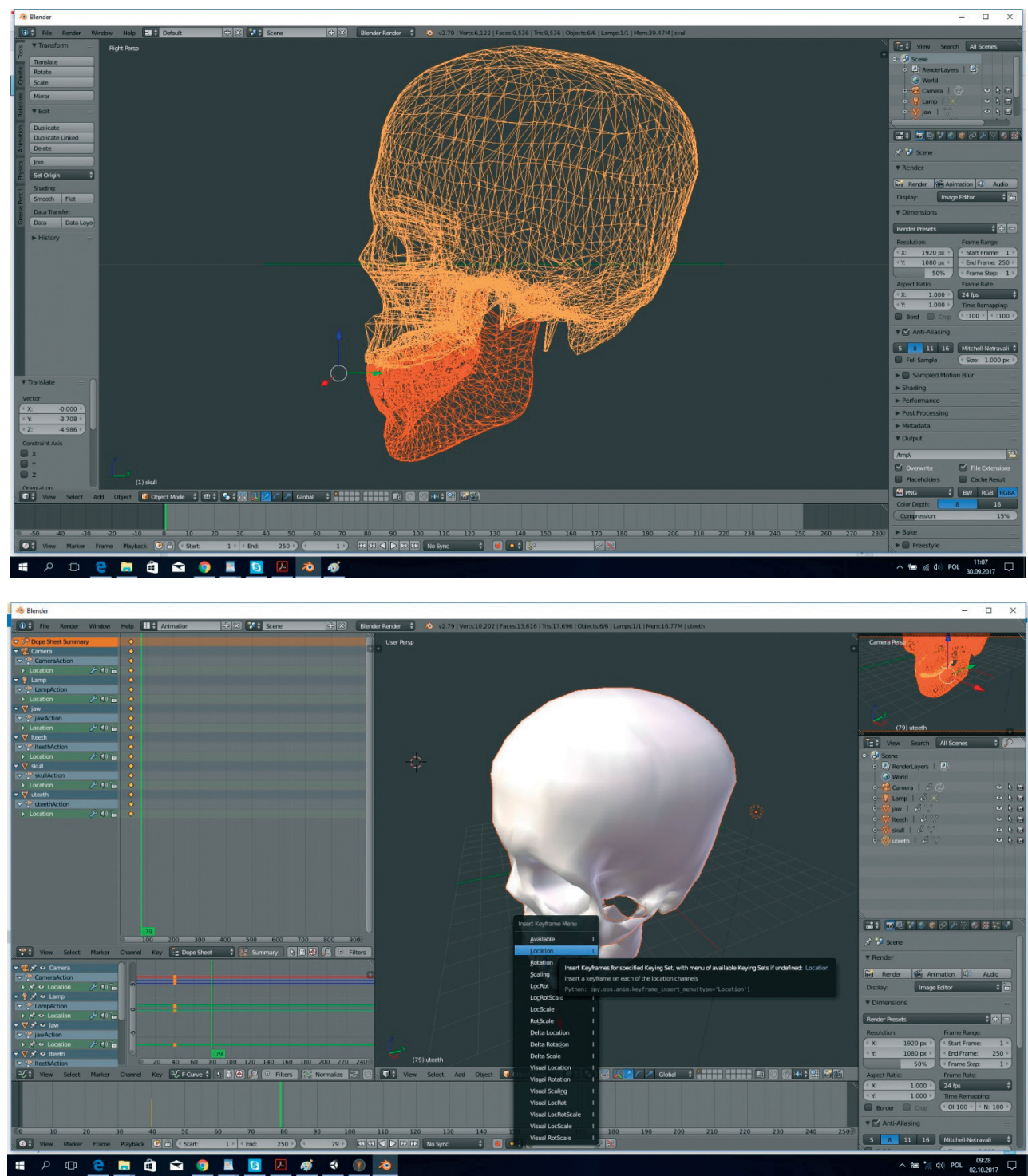

Рис. 3. Обработка в Blender импортированной модели из BodyParts3D:

а) сеточная модель; б) создание анимации

Интерактивные приложения с использованием анатомических структур могут активно опираться на использование следующих составляющих Unity.

Физический движок Unity является одним из наиболее важных компонент для создания эффектов виртуальной реальности. Для того чтобы объект 3D (в данном случае анатомическая структура) обрабатывался физическим движком, он должен содержать в себе компонент Rigidbody. В дальнейшем это позволяет связать с данным компонентом такие свойства, как масса (в относительных единицах измерения), ускорение перемещения, угловое ускорение, использование гравитации, кинематичности; интерполировать перемещения, указать точность обнаружения столкновений (коллизий).
Со способом обнаружения коллизий связано использование соответствующих компонент физического движка, а именно: BoxCollider следует использовать в случае отслеживания столкновения объектов кубической формы (или близкого к этому), Sphere Collider - сферической, Capsule Collider - в виде капсулы, Mesh Collider - в виде сетки, Terrain Collider - в виде сложной формы поверхности. При этом стоит учитывать, что использование двух последних компонент значительно увеличивает нагрузку на графический процессор, что требует их осторожного использования.

Следующая группа компонент физического движка связана с использованием соединений физических тел. При этом поддерживаются такие важные для анатомических структур 
типы соединений: подвижные (Hinge Joint), фиксированные (Fixed Joint), упругие (Spring Joint) и соединения с произвольной конфигурацией (Configurable Joint).

Внешнюю приложенную силу следует моделировать посредством компонента Constant Force.

Использование анимационной системы Mecanim. Unity обладает изощрённой анимационной системой Месаnim. Она обеспечивает простоту схемы управления и настройки анимации для всех элементов Unity, включая объекты, их части и свойства; поддержку импортированных анимационных клипов и анимаций, созданных внутри Unity; перенацеливание гуманоидных анимаций - способность применять анимации из одной модели персонажа на другую; упрощенный процесс выравнивания анимационных клипов; удобный предварительный просмотр анимационных клипов, переходы и взаимодействия между ними. Это позволяет аниматорам работать более независимо от программистов, отлаживать анимации ещё до наложения программного кода; управлять сложными взаимодействиями между анимациями, используя визуальное программное средство; также позволяет анимирование различных частей тела с разной логикой, многоуровневость.

Как правило, с каждым 3D-объектом связан ряд анимаций, созданных ранее. Управление выполнения таких анимаций осуществляется с помощью специально созданного компонента Animator Controller. Переключения между анимациями происходят при наступлении в программе определенных событий. Например, можно запустить анимацию оборачивания анатомической структуры, нажав клавишу г. Даже если существует только один анимационный клип, он всё равно должен быть помещён в компонент Animator Controller для его использования в Game Object.

Контроллер управляет состояниями разных анимаций и переходами между ними так называемой машиной состояний, которую можно себе представить как блок-схему или простую программу, написанную на визуальном языке программирования внутри Unity. Структура контроллера Animator Controller создаётся, представляется и изменяется в окне Animator Window.

Каждый Animator Controller определяет входное состояние под названием Entry, выходное состояние - Exit. Далее каждому клипу, связанному с объектом (компоненты Animation), отвечает своё состояние (соответственно блок в блок-схеме

в блок-схеме окна Animator). Переходы между состояниями (блоками, анимациями) создаются с помощью визуального инструментария Мecanim на блок-схеме с помощью контекстного меню Make transition.

Каждый переход между анимациями представляется в качестве объекта, который настраивается в окне инспектора объектов. При этом для управления переходами используются специально создаваемые во вкладке Parameters окна Animator параметры. Такие параметры могут быть четырёх типов: Float, Int, Bool, Trigger. Причём при употреблении булевского параметра типа Trigger его значение каждый раз переключается при использовании его для перехода.

Создав параметры и определив с их помощью условия выполнения переходов между анимациями в инспекторе объектов для переходов (панель Conditions) далее созданный механизм управления анимациями может быть использован в программном коде на языке С\#. Например, так обрабатывается нажатие клавиш клавиатуры для вызова анимаций:

$\mathrm{r}$ - оборачивания, s - масштабирования:

using UnityEngine;

using System.Collections;

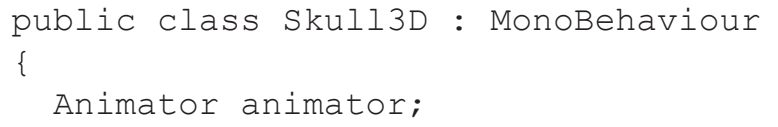




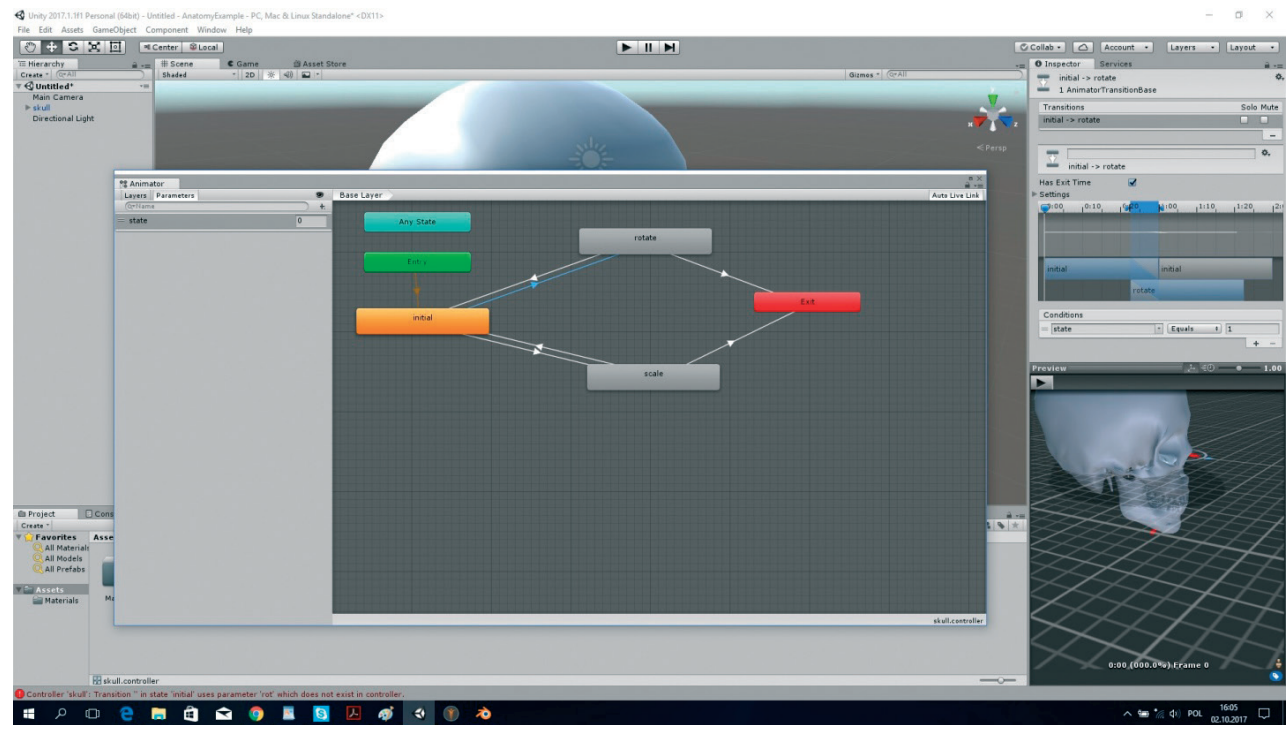

Рис. 4. Создание и управление анимациями с помощью системы Mecanim

Анимация может быть также вызвана при сталкивании с другим определенным объектом (возникновение коллизии):

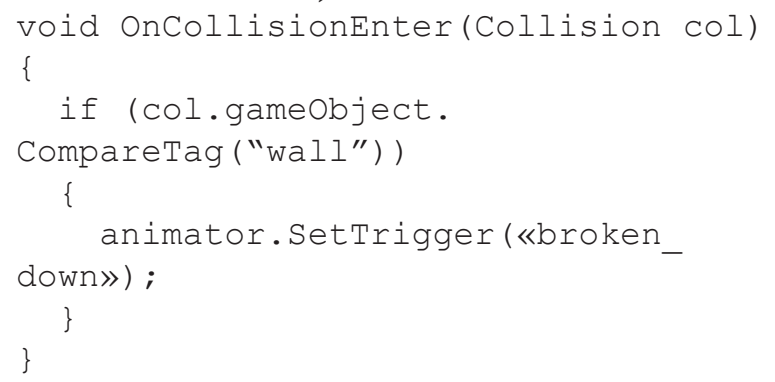

определенным тегом wall. Создавая и используя таким образом блок-схемы системы Mесаnim, достигаются весьма сложные механизмы управления анимациями, связанными с 3D-моделями (рис. 4).

Создание интерактивности с помощью триггеров. Под триггерами, как правило, имеют в виду очень общие сущности (возможны даже функции, программный код и т. д.), с помощью которых осуществляется управление выполнения программы через какие-то взаимодействия (интерактивность). В случае 3D-графики Unity понятие триггеров используется для свойства компонент типа Collider физического движка. Установление данного свойства для компонент позволяет далее обрабатывать события, связанные с контактами с данным объектом: при входе (столкновении) в данный объект, при нахождении в данном объекте, при выходе из объекта. Такие действия для обеспечения интерактивности могут быть обработаны в соответствующих методах:

void OnTriggerEnter (Collider collider)

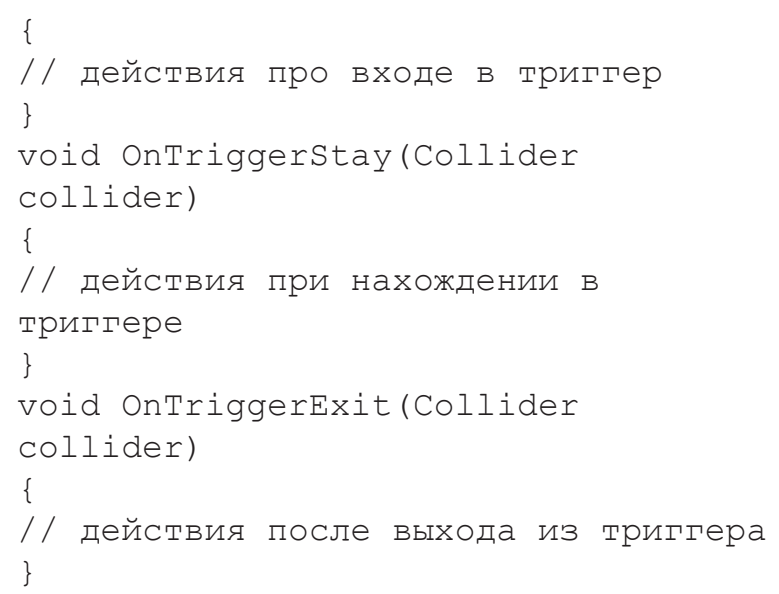

Выводы. Таким образом, в работе проведено исследование проблем, возникающих при обучении студентов-медиков на физических моделях, и предложен подход для разработки интерактивного программного обеспечения, использующего компьютерную графику 3D в медицинском образовании.

Укажем, что указанный порядок разработки ориентирован на использование уже приготовленных заранее моделей анатомических структур, создаваемых, например, на основе МРТ-изображений и представленных в разных репозиториях — как коммерческих, так и свободно распространяемых (например, BodyParts3D). Далее процесс состоит в использовании бесплатного программного обеспечения, что, по мнению авторов, способствовало бы широкой разработке интерактивных обучающих программ в медицинских вузах. 
Возможность лицензирования предложенного программного обеспечения

\begin{tabular}{|l|c|c|c|}
\hline \multicolumn{1}{|c|}{ Этап работы } & $\begin{array}{c}\text { Используемое } \\
\text { программное } \\
\text { обеспечение }\end{array}$ & Компания-разработчик & Лицензия \\
\hline $\begin{array}{l}\text { Использование 3D-моделей анатомиче- } \\
\text { ских структур, разработанных в проекте } \\
\text { Anatomography }\end{array}$ & BodyParts3D & Anatomography & Free open-source \\
\hline $\begin{array}{l}\text { Получение сетевых моделей человека } \\
\text { с возможностью модификации }\end{array}$ & MakeHuman & The MakeHuman team & AGPL \\
\hline $\begin{array}{l}\text { Импортирование и обработка 3D-модели, } \\
\text { создание анимаций }\end{array}$ & Blender & Blender Foundation & GNU GPL \\
\hline $\begin{array}{l}\text { Создание интерактивного приложения } \\
\text { с управляемыми анимациями }\end{array}$ & Unity Technologies & Unity Technologies & Proprietary \\
\hline
\end{tabular}

Авторы считают, что использование современных лицензированных средств разработки (табл. 1), которые получили сегодня популярность, в первую очередь, при разработке развлекательных программных продуктов (компьютерных игр), имеет ряд преимуществ при использовании для разработки приложений компьютерной графики в медицинском образовании: во-первых, направленность на обработку сложных сеточных моделей, 3D-визуализацию, создание эффектов, интерактивное управление анимациями; во-вторых,

\section{Литература.}

1. Anatomical models: a digital revolution / J. R. Fredieu, J. Kerbo, M. Herron [et al.] // Med. Sci. Educ. — 2015. — Vol. 25, №. 2. - P. 183-194.

2. AnatomyBrowser: a novel approach to visualization and integration of medical information / P. Golland, R. Kikinis, M. Halle [et al.] // Comput. Aided Surg. —1999. — Vol. 4, №. 3. — P. 129-143.

3. Becker B. W. Digital learning object repositories / B. W. Becker // Behav. Soc. Sci. Libr. - 2010. Vol. 29. - P. 86-88.

4. Carolan J. Using representations for teaching and learning in science / J. Carolan, V. Prain, B. Waldrip // Teaching Science: The Journal of the Australian Science Teachers Association. - 2008. - Vol. 54, №. 1. - P. 18-23.

5. Douglass C. Plastination: preservation technology enhances biology teaching / C. Douglass, R. Glover // Am. Biol. Teach. (Natl. Assoc. Biol. Teach.). — 2003. Vol. 65, №. 7. - P. 503-510.

6. Hopkins R. Exploring the changing learning environment of the gross anatomy lab / R. Hopkins, G. Regehr, T. D. Wilson // Acad. Med. — 2011. — Vol. 86, №. 7. P. 883-888.

7. Hopwood N. Model politics / N. Hopwood // Lancet. 2008. — Vol. 372, №. 9654. — P. 1946-1947. наличие достаточного количества специалистовпрограммистов, владеющими данными технологиями; в-третьих, достаточная отлаженность данных программных сред (при разработке проектов Blender, Unity задействовано много высококлассных программистов, проекты поддерживаются достаточно длительное время, сегодня разработано большое количество версий программных продуктов с целью усовершенствования и исправления ошибок).

8. How useful is plastination in learning anatomy? / R. M. Latorre, M. P. García-Sanz, M. Moreno [et al.] // J. Vet. Med. Educ. — 2007. — Vol. 34, №. 2. P. 172-176.

9. Maerker A. Florentine anatomical models and the challenge of medical authority in late-eighteenth-century Vienna / A. Maerker // Stud. Hist. Phil. Biol. Biomed. Sci. - 2012. — Vol. 43, №. 3. - P. 730-740.

10. Manson A. A recommended workflow methodology in the creation of an educational and training application incorporating a digital reconstruction of the cerebral ventricular system and cerebrospinal fluid circulation to aid anatomical understanding / A. Manson, M. Poyade, P. Rea // BMC Med. Imaging. — 2015. — Vol. 15. — P. 44.

11. Marković D. Development of anatomical models chronology / D. Marković, B. Marković-Živković // Acta Med. Medianae. — 2010. — Vol. 49, №. 2. — P. 56-62.

12. Marks S. C. Jr. The role of three-dimensional information in health care and medical education: the implications for anatomy and dissection / S. C. Marks Jr // Clin. Anat. 2000. — Vol. 13, № 6. — P. 448-452.

13. Nieder G. L. Using quicktime virtual reality objects in computer-assisted instruction of gross anatomy: Yorick - the VR skull / G. L. Nieder, J. N. Scott, 
M. D. Anderson // Clin. Anat. - 2000. — Vol. 13, № 4. - P. 287-293.

14. Russell K. F. Ivory anatomical manikins / K. F. Russell // Med. Hist. — 1972. — Vol. 16, № 2. - P. 131-142.

15. Schwartz D. Spatial representations and imagery in learning / D. Schwartz, J. Heiser // R. Sawyer (ed.) // The Cambridge handbook of the learning sciences. Cambridge : Cambridge University Press. - P. 283-289.

16. Share and enjoy: anatomical models database generating and sharing cardiovascular model data using web services / E. Kerfoot, P. Lamata, S. Niederer [et al.] // Med. Biol. Eng. Comput. — 2013. — Vol. 51, № 11. — P. 1181-1190.

17. Stahl G. Computer-supported collaborative learning / G. Stahl, T. Koschmann, D. Suthers / R. Sawyer (ed.) // The Cambridge handbook of the learning sciences. Cambridge : Cambridge University Press. - P. 409-426.

18. The evolution of anatomical illustration and wax modelling in Italy from the 16th to early 19th centuries / A. Riva, G. Conti, P. Solinas, F. Loy // J. Anat. 2010. — Vol. 216, № 2. — P. 209-222.

19. The evolution of learning object repository technologies: portals for on-line objects for learning / G. Richards, R. McGreal, M. Hatala, N. Friesen // J. Dist. Educ. 2002. — Vol. 17, № 3. - P. 67-79.

\section{References.}

1. Fredieu, J. R., Kerbo, J., Herron, M., Klatte, R. \& Cooke, M. (2015). Anatomical models: a digital revolution. Med. Sci. Educ., 25(2), 183-194. doi 10.1007/s40670-0150115-9.

2. Golland, P., Kikinis, R., Halle, M., Umans, C., Grimson, W., Shenton, M. E., \& Richolt, J. A. (1999). AnatomyBrowser: a novel approach to visualization and integration of medical information. Comput. Aided Surg., 4(3), 129-143. doi: 10.3109/10929089909148168.

3. Becker, B. W. (2010). Digital learning object repositories. Behav. Soc. Sci. Libr., 29, 86-88.

4. Carolan, J., Prain, V., \& Waldrip, B. (2008). Using representations for teaching and learning in science. Teaching Science: The Journal of the Australian Science Teachers Association, 54(1), 18-23.

5. Douglass, C., \& Glover, R. (2003). Plastination: preservation technology enhances biology teaching. Am. Biol. Teach. (Natl. Assoc. Biol. Teach.), 65(7), 503-510. doi: $10.2307 / 4451549$

6. Hopkins, R., Regehr, G., \& Wilson, T. (2011). Exploring the changing learning environment of the gross anatomy lab. Acad Med., 86(7), 883-888. doi: 10.1097/ ACM.0b013e31821de30f.

7. Hopwood, N. (2008). Model politics. Lancet, 372(9654), 1946-1947.

8. Latorre, R. M, García-Sanz, M. P., Moreno, M., Hernández, F., Gil, F., López, O. ...Henry, R. W. (2007). How useful is plastination in learning anatomy? J. Vet Med Educ., 34(2), 172-176.
9. Maerker, A. (2012). Florentine anatomical models and the challenge of medical authority in late-eighteenth-century Vienna. Stud. Hist. Phil. Biol. Biomed. Sci., 43(3), 730-740. doi: 10.1016/j.shpsc.2012.02.005

10. Manson, A., Poyade, M., \& Rea, P. (2015). A recommended workflow methodology in the creation of an educational and training application incorporating a digital reconstruction of the cerebral ventricular system and cerebrospinal fluid circulation to aid anatomical understanding. BMC Med. Imaging, 15, 44. doi: 10.1186/s12880-015-0088-6.

11. Marković, D., \& Marković-Živković, B. (2010). Development of anatomical models - chronology. Acta Med. Medianae, 49(2), 56-62.

12. Marks S., Jr (2000). The role of threedimensional information in health care and medical education: the implications for anatomy and dissection. Clin. Anat., 13(6), 448-452. doi: 10.1002/1098-2353(2000)13:6<448::AIDCA10>3.0.CO;2-U.

13. Nieder, G., Scott, J., \& Anderson, M. D. (2000). Using quicktime virtual reality objects in computer-assisted instruction of gross anatomy: Yorick - the VR skull. Clin. Anat., 13(4), 287293. doi: 10.1002/1098-2353(2000)13:4<287::AIDCA9>3.0.CO;2-L.

14. Russell, K. F. (1972). Ivory anatomical manikins. Med. Hist., 16(2), 131-142.

15. Schwartz, D., \& Heiser, J. (2006). Spatial representations and imagery in learning. In R. Sawyer (Ed.). The Cambridge handbook of the learning sciences (pp. 283289). Cambridge: Cambridge University Press.

16. Kerfoot, E., Lamata, P., Niederer, S., Hose, R., Spaan, J., \& Smith, N. (2013). Share and enjoy: anatomical models database - generating and sharing cardiovascular model data using web services. Med. Biol. Eng. Comput., 51(11), 1181-1190. doi: 10.1007/s11517012-1023-4.

17. Stahl, G., Koschmann. T., Suthers, D. (2006). Computersupported collaborative learning. In R. Sawyer (Ed.). The Cambridge handbook of the learning sciences (pp. 409-426). Cambridge: Cambridge University Press.

18. Riva, A., Conti, G., Solinas, P., \& Loy, F. (2010). The evolution of anatomical illustration and wax modelling in Italy from the 16th to early 19th centuries. J. Anat., 216(2), 209-222. doi: 10.1111/j.14697580.2009.01157.

19. Richards, G., McGreal, R., Hatala, M., \& Friesen, N. (2002). The evolution of learning object repository technologies: portals for on-line objects for learning. J. Dist. Educ., 17(3), 67-79. 